\title{
A STUDY OF NAIL CHANGES IN VARIOUS DERMATOSIS IN PUNJAB, INDIE
}

\author{
by Neerja Puri, Tejinder Kaur
}

comment:

Dr. Leonel Fierro, Dr. Alexandro Bonifaz

Our Dermatol Online. 2012; 3(3): 171

Date of submission: 01.04.2012 / acceptance: 06.04.2012

Conflicts of interest: None

The article by Drs Puri and Kaur communicate in a clear and consistent way the various changes that can be found on the nails and their relationship with other dermatoses. As indicated in the beginning, the nails are a ,window" to suspect the presence of other associated diseases and to establish diagnostic proposals. Understanding the nail as a ,functional unit”, the range of entities that the clinician faces is extremely varied, hence the daily practice of dermatologists may encounter inflammatory, autoimmune, infectious, pharmacologic, traumatic and tumoral conditions. Moreover nails reflect signs associated with other dermatoses or with systemic diseases that have no impact to another cutaneous level. The authors obtain a sufficient population to identify some characteristics associated with nail pathology described as: age range, occupation, number of nails involved, diagnosis and some specific findings in states such as nail psoriasis. Based on our experience it is worth noting the contrast at two points in particular, the first is on the prevalence of onychomycosis in our population that exceeds $50 \%$ of the cases and is the most common pathology at this level, being the first complaint of nail disease. The second observation is that we have identified that age group with the greatest impact of nail pathology is over 40 years and differs from that discussed in the communication. This may be because in our patients, high rates of associated systemic diseases such as diabetes mellitus and other local processes such as vascular illness, heart disease, tinea pedis, poor hygiene and bad nutrition are factors that may impact adversely on their health, increasing the number of cases with these characteristics. Auxiliary diagnostic techniques currently available as dermatoscopy, laboratory tests and biopsies, are useful tools for more accurate conclusions in the pathology of the nail.
Finally there will note the importance of tackling the nail apparatus associated tumors of which the variety of benign strain injuries require experience to establish the diagnostic suspicion and especially melanonychia should be approached with grater care while maintaining the intention of discarding the presence of a malignant melanoma of the serious risks involved. This paper invites in a friendly and clear form to the comprehensive approach on nail diseases, taken into account comorbidities and other conditions. As the article concludes it is important to successful integration and diagnostic correlation, so we can offer the best chance for patients to receive better quality care.

\section{Correspondence:}

Dr. Leonel Fierro, Dr. Alexandro Bonifaz

Dermatology Service and Mycology Department, Hospital General de México O.D.

México City, México.

E-mai: Dr. Alexandro Bonifaz: a bonifaz@yahoo.com.mx

Copyright by Leonel Fierro, et al. This is an open access article distributed under the terms of the Creative Commons Attribution License, which permits unrestricted use, distribution, and reproduction in any medium, provided the original author and source are credited. 